\title{
BMJ Open Suicidal behaviours and moderator support in online health communities: protocol for a scoping review
}

\author{
Amanda Perry (D) , ${ }^{1,2}$ Andrea Lamont-Mills, ${ }^{3}$ Carol du Plessis, ${ }^{3}$ Jan du Preez, ${ }^{1}$ \\ Denise Pyle ${ }^{3}$
}

To cite: Perry A, LamontMills A, du Plessis C, et al. Suicidal behaviours and moderator support in online health communities: protocol for a scoping review. BMJ Open 2020;10:e034162. doi:10.1136/ bmjopen-2019-034162

- Prepublication history and additional material for this paper are available online. To view these files, please visit the journal online (http://dx.doi. org/10.1136/bmjopen-2019034162).

Received 08 September 2019 Revised 19 December 2019 Accepted 30 December 2019

A) Check for updates

C Author(s) (or their employer(s)) 2020. Re-use permitted under CC BY-NC. No commercial re-use. See rights and permissions. Published by BMJ.

${ }^{1}$ School of Psychology and Counselling, University of Southern Queensland, Toowoomba, Queensland, Australia

${ }^{2}$ School of Community Studies, Unitec, Auckland, New Zealand

${ }^{3}$ School of Psychology and Counselling, University of

Southern Queensland, Ipswich, Queensland, Australia

Correspondence to

Amanda Perry;

amanda.perry@usq.edu.au

\section{ABSTRACT}

Introduction Suicidal ideation and suicidal behaviours are common yet complex mental health presentations that can pose significant challenges for health professionals. The inability to accurately predict the individuals who may move from experiencing suicidal ideation and associated behaviours, to completing suicide, presents one such challenge. This can make it difficult to provide interventions and support to those most in need. Online health communities are one possible source of support for individuals who experience suicidal ideation and behaviours. These communities are becoming an increasingly popular way of accessing support, often with life-saving consequences. Within online communities, support is offered by various individuals including, in some instances, health professionals from various backgrounds, who work as online health community moderators. Given the growth of online communities and the increasing number of health professionals working as moderators, this scoping review seeks to map the literature that has focused on health professionals working as online community moderators, who interact with members experiencing suicidal ideation and behaviours. Mapping the existing literature offers benefits to both research and practice by identifying gaps in the research and providing a beginning knowledge base of current practice that can inform the training and development of health professionals working as community moderators. Methods and analysis This scoping review will follow the methodological framework of Arksey and 0'Malley, later adapted by Levac et al. To ensure appropriate rigour, this protocol uses the 20-item Preferred Reporting Items for Systematic Reviews and Meta-Analyses and extension for Scoping Reviews. Literature will be identified using a search strategy developed in consultation with a specialist research librarian at the university where the researchers are employed. Ten multidisciplinary databases will be independently searched by two researchers, and both researchers will screen for inclusion, and undertake the data extraction. The first author will perform a quality assessment of the articles that are selected for inclusion. A second researcher will complete a random audit of $20 \%$ of the included articles to assess for quality and suitability in answering the research questions. The first author will complete the analysis and synthesis of the data. A numerical and narrative synthesis of the included studies will be provided.

\section{Strengths and limitations of this study}

To our knowledge, this scoping review will be the first to review and summarise research that has focused on health professionals working as online community moderators who support individuals experiencing suicidal behaviours (suicidal ideation, suicidal behaviours and suicide attempt). It will provide a baseline for future research

- Strengths of this study include the use of an established scoping review methodology, a rigorous search strategy developed in consultation with a specialist research librarian, a systematic study selection carried out by two researchers, and a quality assessment of included literature.

- The scoping review will focus on peer-reviewed articles and findings will be limited to articles that are written in English (or translated into English).

Ethics and dissemination The scoping review has been deemed as being exempt from ethical review as no data will be collected from human participants. The results of the scoping review may be published in a peer-reviewed journal, thesis, presented at relevant conferences, and shared with relevant knowledge users.

\section{INTRODUCTION}

Suicidal behaviours are a significant cause of death and disability worldwide, with close to 800000 people dying by suicide every year. ${ }^{1}$ Despite a growing awareness of the need for suicide prevention, suicide remains a serious public health concern. ${ }^{1}$ While there are publicly available statistics for completed suicides, the same cannot be said where suicide is attempted but not completed, as many suicide attempts are not reported to health professionals. Despite this lack of data, it is estimated that more people attempt suicide than die by suicide. ${ }^{1}$ For this reason, it is estimated that the number of people who are impacted by suicidal behaviours is far greater than the 800000 recorded suicides. ${ }^{1}$ Advances in technology have impacted on suicidal behaviours, in that individuals with 
internet capability can access a range of support and content that can positively and negatively influence suicidal ideation and suicidal behaviours. ${ }^{2}$ For example, access to online support forums are likely to help keep individuals safe as they can connect to peer or professional support in times of need, conversely, individuals can also access content that may promote suicidal behaviours. ${ }^{3}$

Online health communities are internet-based platforms that have become increasingly popular due to their ability to facilitate the sharing of information, advice and support, which can be especially important for individuals who are experiencing suicidal behaviours and therefore may be at risk of serious harm or death. ${ }^{3}$ There is a lack of research that specifically measures the effectiveness of online support health communities. However, the effectiveness and usefulness of these forums for members can be inferred from the growing membership in these forums as well as member retention within these communities. $^{3}$

When considering how to intervene and prevent suicide, it is important to distinguish between the separate, but interconnected, constructs of suicidal ideation, suicidal behaviours or attempts and suicide. Suicidal ideation consists of thoughts about how to kill oneself, which can range from fleeting thoughts, to extensive considerations and detailed plans. ${ }^{4}$ Suicidal behaviours and suicide attempts are deliberate and consciously selfdestructive where the intent is to kill oneself, ${ }^{5}$ and suicide is when intentional death occurs. ${ }^{1}$ Most individuals who experience suicidal ideation do not act on the thoughts or carry them through to their conclusion. ${ }^{2}$ Therefore, while suicidal ideation can place an individual at risk for engaging in suicidal behaviours, suicidal ideation in isolation is not necessarily a high-risk marker for a future suicide attempt.

According to Klonsky and May's ${ }^{5}$ Three Step Theory of Suicide, connectedness plays a critical role in whether an individual moves from ideation towards suicidal behaviours, including a suicide attempt. The first step towards movement begins with psychological or emotional pain and a sense of hopelessness. ${ }^{5}$ If an individual who is experiencing suicidal thoughts has hope that their situation may improve, and that the pain can be diminished, the individual is likely to work towards reducing the pain they feel, rather than consider attempting suicide. ${ }^{5}$ An individual, who experiences psychological pain combined with a sense of hopelessness, is more likely to experience suicidal ideation and be more at risk of moving towards an attempt. ${ }^{5}$

The second step towards a suicide attempt occurs when pain exceeds connectedness. ${ }^{5}$ Connectedness refers to the connection with other people, interests, roles, projects or a sense of meaning that gives one's life purpose. When an individual experiences pain and hopelessness and considers suicide, they are believed to be experiencing moderate suicidal ideation. This does not mean the individual is moving towards suicide, provided their sense of connectedness remains greater than their pain. ${ }^{5}$ However, when their pain overwhelms any sense of connectedness, suicidal ideation is likely to become stronger, and may result in the individual actively considering ending their life, or being on the move towards suicide. ${ }^{5}$

The third step reflects the progression from ideation to attempt and requires individuals to have the capacity to make an attempt on their own life, by overcoming the natural human instinct of fearing death. ${ }^{5}$ An individual is more likely to attempt suicide when they have overcome their fear of death, and they experience a sense of pain and hopelessness that overwhelms any sense of connectedness. ${ }^{5}$ It is at this point that an individual may move towards an attempt.

Individuals struggling with suicidal ideation and suicidal behaviours are traditionally seen by health professionals working in face-to-face settings. While professionals are well positioned to provide support, barriers, such as social stigma ${ }^{6}$ and negative perceptions regarding suicide, can prevent individuals from accessing and engaging in support. ${ }^{5}$ It is acknowledged that not all individuals who seek in-person support receive it, for reasons such as not meeting set assessment criteria, a shortage of resources and wait list times. A lack of suitable support services, particularly for those in rural settings, combined with other barriers such as privacy concerns, time requirements, financial costs, distance and transport required to access support, are further reasons why individuals may not be willing or able to engage with in-person psychological support. ${ }^{2}$ For individuals unable or unwilling to engage with face-to-face support, online health communities offer an easily accessible alternative source of support. $^{78}$

Online health communities typically include a large element of peer support, where members use their previous experiences and resulting insights to offer support. Peer support refers to people's natural tendency to seek support and advice from informal social sources in their immediate environment. ${ }^{9}$ A central tenet of peer support is the commonality of experience between the peers engaged in the supportive interactions. Peer support differs from professional support in that the interactions between peers are voluntary, flexible and informal. ${ }^{10}$

Online health communities can follow different models of support, with one such example being forums that exclusively offer peer interactions without moderation from either peers or professionals. Alternatively, there are forums where peers within the online community fulfil the roles and functions of moderators. In addition, some peer support forums are overseen by professional moderators who either hold formal tertiary-level qualifications or have completed in-house moderator training. Professional moderators can undertake administrative functions such as editing content, and guiding members with the features and functions of the forum, as well as providing professional support to members who may be at risk of engaging in suicidal behaviours. $^{7}$ 
Atanasova $e t a l^{7}$ argue that although online health communities are increasingly becoming the focus of health research, this research has typically focused on forum users and not on forum moderators, whether these are professional or peer moderators. This is despite online health communities providing a new way for health professionals and clients (online community members) to interact with one another. ${ }^{11}$ The increasing use of online health communities as a means of gaining professional support ${ }^{12}$ makes it crucial for health providers and researchers to gain a better understanding of moderator practices in these spaces. ${ }^{13}$ This is due to the traditional face-to-face communication practices of health professionals requiring adaptation in the online environment, ${ }^{14}$ as communication in these spaces is asynchronous, and devoid of non-verbal cues such as body language and movement, details of dress and nuances of the voice. Understanding how health professionals who are moderators offer support to those experiencing suicidal ideation or engaging in suicidal behaviours (including a suicide attempt) is important, given the 24 hours availability and relatively instant nature of moderator support, which can reduce risk to life. Furthermore, the ability of moderators to reach and support a larger number of vulnerable individuals, when compared with individual face-to-face health professionals, makes understanding what constitutes effective moderation practices essential in replicating these practices across online communities. Moderator support is also important as it can be the first professional interaction an individual may have with regard to their mental health. This is significant as the quality of the interaction may influence whether an individual then reaches out for support in their immediate setting. Understanding how moderators interpret, make sense of and then respond to members at risk allows healthcare professionals to further capitalise on the opportunities for positive and potentially life-saving support that are offered by online communities. ${ }^{3}$

The findings from this review will provide a synthesis of the research that has focused on professional moderators who work with members experiencing suicidal ideation, suicidal behaviours, or engaging in a suicide attempt. Currently, there is no systematic review of the literature regarding professional moderators, and therefore, there is no clear understanding of what research has been completed, what research needs to be undertaken and where research needs to focus in the future. The finding of this review will offer implications for practice in that it will provide an evidence base on which organisations can train online moderators.

\section{METHODS}

This review will follow the six-stage scoping review methodological framework proposed by Arksey and O'Malley, ${ }^{15}$ which has been further developed by Levac et al. ${ }^{16}$ The six stages are: (1) identifying the research question; (2) identifying the relevant literature; (3) study selection;
(4) charting the data; (5) collating, summarising and reporting the data; and (6) consultation with knowledge users of online community forums.

\section{Patient and public involvement}

Patients and the public were not involved in the writing of this scoping review protocol.

\section{Stage 1: identifying the research questions}

The aim of this scoping review is to identify what is empirically known about health professionals working as online health community moderators. It is intended that the findings from this review will inform further studies into the work of online community moderators, in order to achieve a greater understanding of the challenges and complexities of the role, especially when supporting members experiencing suicidal ideation and behaviours. It is anticipated that the findings of this review may be used to inform and enhance the recruitment and training of online community moderators, which can lead to improved service delivery to members of online forums.

To assist in the creation of the study research questions, the broad Population-Concept-Context mnemonic by the Joanna Briggs Institute (JBI) was adopted as a suitable alternative to the Population, Intervention, Comparator and Outcome mnemonic for systematic reviews. ${ }^{17}$

\section{Population}

There is one population associated with this research study; online health forum moderators. In the context of this study, a moderator is a qualified healthcare professional who is employed to oversee the content and interactions of an online health community. This moderator will intervene in the forum and interact with members where necessary to ensure their safety. As scoping reviews are an iterative process, the definition of professional moderator and what it means to be qualified may become more clearly defined as a result of the search.

\section{Concept}

Identifying what is known about health professionals working as moderators in online forums where members freely post about suicidal ideation, suicidal behaviours, self-harm and non-suicidal self-injury (NSSI).

\section{Context}

No geographical limitations will be placed on the literature. This is due to suicide and associated behaviours being a global health issue. Furthermore, while the head office of a community forum may be physically located in one country, it is not uncommon for membership access to be available to individuals in other countries, thus making some online community forums international support providers.

To meet these aims the review will be guided by the following questions:

1. What do we know from the existing literature about online mental health moderators who work with suicidal community members? 
2. What methodologies have been used to gain this knowledge?

3. What are the limitations of the research?

4. What are the research gaps?

\section{Stage 2: identifying relevant studies}

The search strategy was iteratively developed in consultation with a specialist research librarian at the university where the researchers are employed. To ensure a comprehensive search of the health sciences literature the following electronic databases will be searched:

- CINAHL with full text, PsycINFO, PsycArticles, Psychology and Behavioral Sciences Collection, Academic Search Ultimate, Health Source: Nursing/ Academic Edition and Sociology Source Ultimate. All of these are located within EBSCOhost.

- ScienceDirect.

- Medline.

- SAGE Journals.

- Taylor and Francis Online.

The search strategy will include subject headings, keywords and related terms for the concepts of suicide (including suicidal ideation, suicidal behaviours and suicide attempts), moderator or facilitator, online community, online health forum or online forum. The search terms for suicidal ideation, behaviours and attempt include 'suicide, 'self-harm' and NSSI. Self-harm and NSSI will be included in the search terms as often the behaviours associated with self-harm and NSSI are classified as suicidal ideation and behaviours, or the deliberate desire to end one's life. For this reason, the inclusion of the terms self-harm and NSSI will ensure adequate coverage of the literature. In the pilot testing of the search strategy, it was determined that the term 'suicd*' would capture all suicide-related literature, including articles that discuss suicidal ideation, suicidal behaviours and suicide attempts. A detailed search strategy can be found in table 1 .

The search will be limited to English articles or those translated into English. A date restriction will be articles published from 1990 to the day of the search. The initial search results will be collated in the reference management programme EndNote (V.9), where duplicates will be removed at the first stage of review. The search will be independently undertaken by two reviewers, who will seek to resolve discrepancies collaboratively. Where this is not possible, a third reviewer will adjudicate to ensure agreement is achieved.

\section{Stage 3: study selection}

All articles will be independently screened for eligibility, beginning with a title and abstract review, followed by a full-text review. The reference lists of the articles selected for inclusion at the full-text review stage will also be searched to identify any further potential sources. The two reviewers who will undertake the initial literature search will also complete the two subsequent levels of screening.

In order to be included in the review studies must meet the following criteria:

1. Published from 1990 when computer-mediated support first appears in the literature.

2. Peer reviewed to ensure only credible and high-quality studies are included.

3. Written in or translated into English (due to a lack of resources for translating articles), with articles that are not written or translated into English excluded at the beginning.

4. Focused on online health forums where members can post freely about suicidal ideation, suicidal behaviours, previous suicide attempts, self-harm or NSSI.

Table 1 Search strings and limiters for each of the selected databases

\begin{tabular}{lll} 
Database & Search strings & Limiters \\
\hline CINAHL with full text, PsycINFO, PsycArticles, & ("online community" OR "online health community" & Time frame: From 1990 \\
Psychology and Behavioral Sciences & OR "online forum") AND moderator OR facilitator & Language: English \\
Collection, Academic Search Ultimate, Health & AND suicid* OR self harm OR NSSI & \\
Source: Nursing/Academic Edition and & & \\
Sociology Source Ultimate & &
\end{tabular}

ScienceDirect ("online community" OR "online health community" Time frame: From 1990

$\begin{array}{lll}\text { Medline (Web of Science) } & \text { ("online community" OR "online health community" Time frame: From 1990 } \\ \text { OR "online forum") AND moderator AND suicid* OR Language: English } \\ \text { "self harm"OR NSSI }\end{array}$
OR "online forum") AND (moderator OR facilitator) AND ("suicidal ideation" OR suicide OR "self harm" OR NSSI)

Language: English

("online community" OR "online health community" Time frame: From 1990 OR "online forum") AND moderator AND suicid* OR Language: English "self harm"OR NSS AND suicid" OR "self harm" OR NSS

OR "online forum" 4 AND moderator
AND suicid* OR "self harm" OR NSS 
5. Participants included in the studies must be qualified healthcare professionals who work as moderators. The qualifications of the professionals would be indicated in the article by listing the professions of the moderators or stating that they are qualified. The definition of qualified healthcare professional is very broad and will include any qualified individual, including professions such as counselling, psychology, social work and mental health nursing.

Exclusion criteria include studies focused on peer or volunteer moderators. It is possible that a professional moderator may hold a dual identity as a professional moderator and a peer, in that they are a qualified professional who has similar personal experiences or mental health concerns as the members of the community that they are overseeing. In instances where a professional moderator also identifies as a peer, their formal and professional role as a moderator will be prioritised over the peer identity.
At the end of each review round, the articles selected for review will be compared by the reviewers. Any discrepancies will be resolved by the two reviewers, or by a third reviewer if consensus cannot be not achieved.

\section{Stage 4: data extraction or 'charting the data'}

The data extraction framework presented in figure 1 will be developed by the research team to confirm study relevance and to extract study characteristics. The extracted data will include bibliographic information (such as author, year and location) and study characteristics (aim, design, methodology, participant characteristics, online community description, outcome measures, key findings, conclusions and quality). This form will be reviewed by the research team and pretested before use to ensure that the required information is being captured. As recommended by Levac et al, ${ }^{16}$ the data extraction form will be continually refined in accordance with the nature and extent of the data, as the reviewers become more familiar with the data during the data collection process.

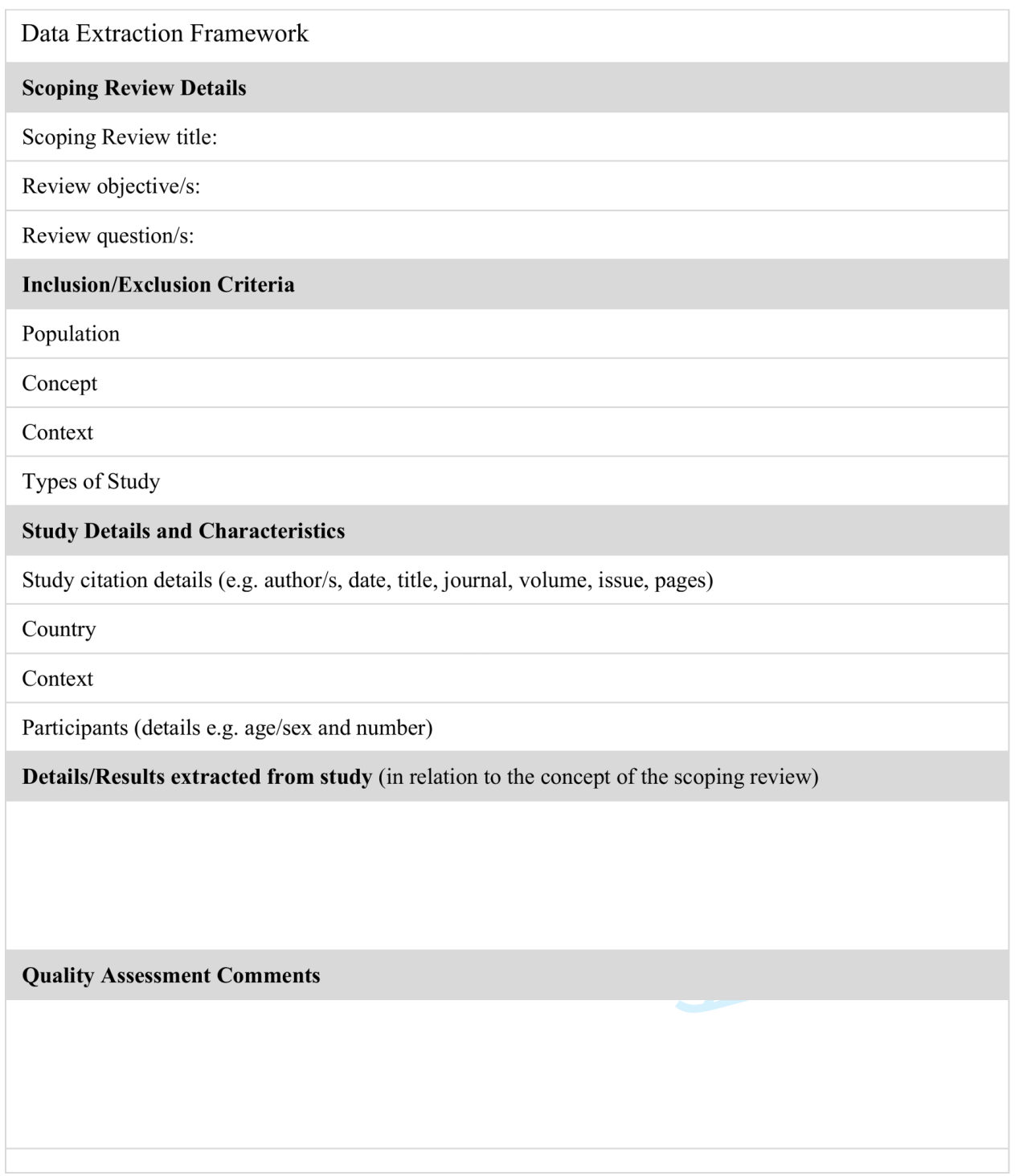

Figure 1 Data extraction framework. Adapted from: Joanna Briggs Institute. ${ }^{21}$ 

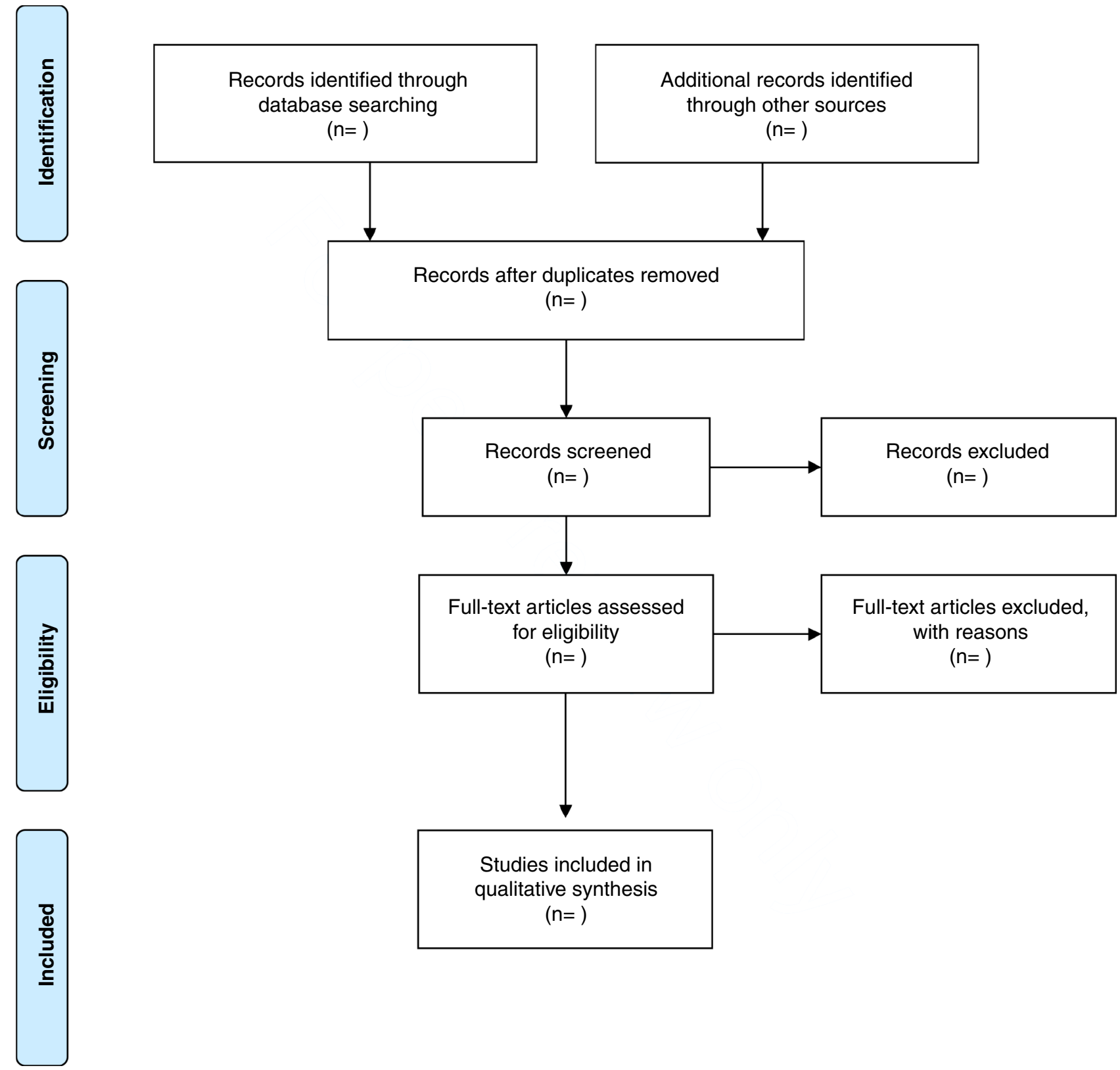

Figure 2 Preferred Reporting Items for Systematic Reviews and Meta-Analyses flow chart. Adapted from: Moher et al. ${ }^{22}$

Consultation on any proposed changes to the extraction form will occur between the two reviewers undertaking the data extraction, with all changes requiring consensus. The data extraction process will be audited for quality and accuracy by sending a random selection $(20 \%$ of the final article number) of extraction article information to an independent reviewer. Any identified issues will be resolved by consensus and a third reviewer will adjudicate if consensus cannot be reached. The process of extraction and sorting will occur in Microsoft Excel, using the data items in the data extraction framework (see figure 1). This will allow for comparison of key items across studies.

As scoping reviews aim to map the existing literature and not to produce a critically synthesised answer to a particular question, a risk of bias assessment is not required for this study. ${ }^{18}$ Assessments of research quality are not typically required by scoping reviews, however, as this scoping review seeks to identify the limitations within the existing moderator literature, an assessment of the quality of the included articles will be performed. ${ }^{15}$ The first author will independently assess the quality of the included articles. The quality assessment process will be audited for accuracy by sending a random selection (20\% of the final article number) of article quality assessment information to an independent reviewer. Any issues that arise will be resolved by consensus and where consensus cannot be achieved, a third reviewer will adjudicate to ensure agreement is achieved. The JBI Appraisal Checklist for Systematic Reviews and Research Syntheses ${ }^{19}$ will be adapted and used for the quality assessment process. The appraisal checklist provides reviewers with a process of critique or appraisal of the research evidence, through 
the assessment of the methodological quality of a study. When appraising a study, reviewers are looking to assess how the possibility of bias has been addressed. ${ }^{19}$ Some examples of the criteria that are used to assess the quality of a study include whether the review question is clearly and explicitly stated and if the inclusion criteria were appropriate for the review question. ${ }^{19}$ Studies that meet more than $80 \%$ of the critical appraisal criteria will be judged to have good methodological quality. Studies that are assessed to have between $50 \%$ and $80 \%$ of the critical appraisal criteria will be deemed to have moderate methodological quality, and studies achieving less than $50 \%$ of the critical appraisal criteria will be judged to have poor methodological quality. Results of the quality assessment undertaken for each included article will be recorded in the data extraction form. It is important to note that unlike systematic literature reviews, studies will not be excluded from this review due to quality assessment outcomes.

\section{Stage 5: collating, summarising and reporting the results}

The Preferred Reporting Items for Systematic Reviews and Meta-Analyses (PRISMA) and extension for Scoping Reviews ${ }^{20}$ checklist for reporting scoping reviews will be used to guide the reporting of the results of this review. It is proposed that the review will combine quantitative and qualitative syntheses to provide an overview of the findings. In order to provide an overview of the breadth of the literature a PRISMA flow chart, presented in figure 2, will be used to report the number of articles present at each stage. A tabular synthesis of the study methodologies, distribution of the studies (geographically), type of online community forums and the characterises of moderators will also be included. A qualitative narrative synthesis will be included and will discuss the limitations of the reviewed studies and identified research gaps.

\section{Stage 6: consultation}

Arksey and $\mathrm{O}^{\prime}$ Malley ${ }^{15}$ suggest that the consultation stage is optional, however, Levac et $a l^{16}$ posit that this stage is imperative to ensuring the methodological rigour of scoping reviews. As part of the consultation process, stakeholders who are subject matter experts will be given the opportunity to become involved in this research by reviewing the preliminary findings, in order to offer their expert perspectives on the findings, make suggestions and offer higher level meanings. For the purpose of this review, the stakeholders selected for consultation will be the service managers of three separate online health community forums. The stakeholders will be contacted via email and invited to review the preliminary findings and share their feedback either via email or a video conferencing meeting. The research questions and outcome measures were informed by the experiences of the first author who is an online community moderator.

\section{LIMITATIONS OF THE PROPOSED REVIEW}

While the search terms and the included databases have been developed to capture all relevant studies, it is noted that the lack of systematic reviews on this topic indicates that there are limited studies that have focused on the substantive area. It is possible that this scoping review may be limited by the small number of studies that are identified and included. This limitation may be mitigated in part by the flexibility of the scoping review design itself. Scoping reviews can be used with a range of data pool sizes and have been proposed as an appropriate design when there is limited data on a topic. ${ }^{15}$ Some literature may also be missed by excluding studies that have not been peer reviewed.

\section{ETHICS AND DISSEMINATION}

To our knowledge, this is the first scoping review to synthesise what is known about health professionals working as online community moderators, in the context of supporting members who are experiencing suicidal ideation and behaviours. This review will identify gaps in the knowledge and research, while also helping to inform the best practice and contribute to the advancement of research and practice on this subject. The results of the scoping review may be published in a peer-reviewed journal, a thesis, presented at relevant conferences, and shared with relevant knowledge users.

Twitter Amanda Perry @mandiperry and Andrea Lamont-Mills @ALamontMills

Acknowledgements The authors gratefully acknowledge the helpful comments of the peer reviewers.

Contributors The design and development of this study was led by AP, who also drafted the protocol. AL-M, CdP, JdP and DP provided guidance to the study conceptualisation and protocol development. All authors have revised all drafts of this manuscript and give approval for the publishing of this protocol manuscript.

Funding The primary author is the recipient of the University of Southern Queensland Research Training Program Stipend Scholarship.

Competing interests None declared.

Patient consent for publication Not required.

Ethics approval This scoping review was exempt from ethics review as no data is being collected from human participants (see page 7 and sections 5.1.22 and 5.1.23 of the Australian National Statement on Ethical Conduct in Human Research).

Provenance and peer review Not commissioned; externally peer reviewed.

Open access This is an open access article distributed in accordance with the Creative Commons Attribution Non Commercial (CC BY-NC 4.0) license, which permits others to distribute, remix, adapt, build upon this work non-commercially, and license their derivative works on different terms, provided the original work is properly cited, appropriate credit is given, any changes made indicated, and the use is non-commercial. See: http://creativecommons.org/licenses/by-nc/4.0/.

ORCID iD

Amanda Perry http://orcid.org/0000-0002-0361-8397

\section{REFERENCES}

1 WHO. Suicide data [Internet]. Available: http://www.who.int/mental_ health/prevention/suicide/suicideprevent/en/ [Accessed 1 Jul 2018].

2 Bell J, Mok K, Gardiner E, et al. Suicide-related Internet use among suicidal young people in the UK: characteristics of users, effects of use, and barriers to offline help-seeking. Arch Suicide Res 2018;22:263-77. 
3 Prescott J, Hanley T, Ujhelyi K. Peer communication in online mental health forums for young people: directional and nondirectional support. JMIR Ment Health 2017;4:e29.

4 Nock MK, Millner AJ, Joiner TE, et al. Risk factors for the transition from suicide ideation to suicide attempt: results from the army study to assess risk and resilience in servicemembers (army STARRS). $J$ Abnorm Psychol 2018;127:139-49.

5 Klonsky ED, May AM. The three-step theory (3ST): a new theory of suicide rooted in the "ideation-to-action" framework. Int J Cogn Ther 2015;8:114-29.

6 Corrigan PW, Sheehan L, Al-Khouja MA, et al. Making sense of the public stigma of suicide. Crisis 2017;38:351-9.

7 Atanasova S, Kamin T, Petrič G. The benefits and challenges of online professional-patient interaction: comparing views between users and health professional moderators in an online health community. Comput Human Behav 2018;83:106-18.

8 Wiggins S, McQuade R, Rasmussen S. Stepping back from crisis points: the provision and acknowledgment of support in an online suicide discussion forum. Qual Health Res 2016;26:1240-51.

9 Xing W, Goggins S, Introne J. Quantifying the effect of informational support on membership retention in online communities through large-scale data analytics. Comput Human Behav 2018;86:227-34.

10 Lekka F, Efstathiou G, Kalantzi-Azizi A. The effect of counsellingbased training on online peer support. Br J Guid Counc 2015;43:156-70.

11 Ali K, Farrer L, Gulliver A, et al. Online peer-to-peer support for young people with mental health problems: a systematic review. JMIR Mental Health 2015;2:e19.

12 Huh J, Kwon BC, Kim S-H, et al. Personas in online health communities. J Biomed Inform 2016;63:212-25.
13 Skea ZC, MacLennan SJ, Entwistle VA, et al. Enabling mutual helping? Examining variable needs for facilitated peer support. Patient Educ Couns 2011;85:e120-5.

14 Oftedal B, Kolltveit Beate-Christin Hope, Graue M, et al. Reconfiguring clinical communication in the electronic counselling context: the nuances of disruption. Nursing Open 2019;6:393-400.

15 Arksey H, O'Malley L. Scoping studies: towards a methodological framework. Int J Soc Res Methodol 2005;8:19-32.

16 Levac D, Colquhoun H, O'Brien KK. Scoping studies: advancing the methodology. Implement Sci 2010;5.

17 Joanna Briggs Institute Reviewer's Manual. Developing the title and question [Internet]. Available: https://wiki.joannabriggs.org/display/ MANUAL/11.2.2+Developing+the+title+and+question [Accessed 28 Aug 2019].

18 Munn Z, Peters MDJ, Stern C, et al. Systematic review or scoping review? Guidance for authors when choosing between a systematic or scoping review approach. BMC Med Res Methodol 2018;18:143.

19 Joanna Briggs Institute Reviewer's Manual. Checklist for systematic reviews and research synthese [Internet]. Available: https://joannabriggs. org/sites/default/files/2019-05/JBI_Critical_Appraisal-Checklist_for_ Systematic_Reviews2017_0.pdf [Accessed 13 Oct 2019].

20 Tricco AC, Lillie E, Zarin W, et al. PRISMA extension for scoping reviews (PRISMA-ScR): checklist and explanation. Ann Intern Med 2018;169:467.

21 Joanna Briggs Institute. Joanna Briggs Institute Reviewer's Manual. [(Internet]). Available: https://wiki.joannabriggs.org/display/MANUAL/ 11.3.7.3+Data +extraction [Accessed 1 Jul 2019].

22 Moher D, Liberati A, Tetzlaff J, et al. Preferred reporting items for systematic reviews and meta-analyses: the PRISMA statement. PLoS Med 2009;6:e1000097. 